\title{
Magnetic domains in spinor Bose-Einstein condensates
}

\author{
Michał Matuszewski, Tristram J. Alexander, and Yuri S. Kivshar \\ Nonlinear Physics Center and ARC Center of Excellence for Quantum-Atom Optics, \\ Research School of Physics and Engineering, Australian National University, Canberra ACT 0200, Australia \\ E-mail: ysk@internode.on.net
}

Received December 14, 2009

\begin{abstract}
We discuss the structure of spin-1 Bose-Einstein condensates in the presence of a homogenous magnetic field. We demonstrate that the phase separation can occur in the ground state of antiferromagnetic (polar) condensates, while the spin components of the ferromagnetic condensates are always miscible, and no phase separation occurs. Our analysis predicts that this phenomenon takes place when the energy of the lowest homogenous state is a concave function of the magnetization. We propose a method for generation of spin domains by adiabatic switching of the magnetic field. We also discuss the phenomena of dynamical instability and spin domain formation.
\end{abstract}

PACS: $03.75 . L m$ Tunneling, Josephson effect, Bose-Einstein condensates in periodic potentials, solitons, vortices, and topological excitations; 05.45.Yv Solitons.

Keywords: Bose-Einstein condensates, homogeneous magnetic field, magnetic domains.

\section{Introduction}

The analysis of the properties of spin domains and magnetic solitons is one of the major topics of the theory of crystalline magnetic structures [1]. However, the recent development in the physics of cold gases gave a birth to an exciting new field where the spinor dynamics and magnetic domain formation are the key ingredients of a new and seemingly different physics which, however, borrows many important results and techniques from the solid state physics. More specifically, the spin degree of freedom of spinor Bose-Einstein condensates (BECs) [2-4] leads to a wealth of new phenomena not possessed by singlecomponent (spin-frozen) condensates. New spin-induced dynamics such as spin waves [3], spin-mixing [5] and spin textures $[3,6]$ have all been predicted theoretically and observed in experiment. The observation of these spindependent phenomena became possible due to the development of optical traps [7] which trap all spin components, rather than just the low-magnetic-field seeking spin states of magnetic traps. However, the effect of an additional small non-zero magnetic field on the condensate in these optical traps was studied even in the seminal theoretical [4] and experimental [2] works. In fact the interplay of spin and magnetic field has been at the heart of some of the most impressive spinor BEC experiments, including the demonstration of spin domains [2], spin oscillations [8] and observation of spin textures and vortices [9].
A spin-1 BEC in a magnetic field is subjected to the well-known Zeeman effect. At low fields the effect is dominated by the linear Zeeman effect, which leads to a Larmor precession of the spin vector about the magnetic field at a constant rate, which is unaffected by spatial inhomogeneities in the condensate [10]. At higher magnetic fields the quadratic Zeeman effect becomes important, and leads to much more dramatic effects in the condensate, such as coherent population exchange between spin components $[8,11,12]$ and the breaking of the single-mode approximation (SMA) $[13,14]$, which assumes that all the spin components share the same spatial density and phase profile. The study of the behavior of a spin-1 condensate in the presence of a magnetic field began with the work of Stenger et al. [2], where the existence of magnetic (spin) domains were predicted and observed in the ground state of a polar ${ }^{23} \mathrm{Na}$ condensate subject to a magnetic field gradient. At the same time, the ground states of both ferromagnetic and antiferromagnetic (polar) condensates in homogenous magnetic field were found to be free of spin domains in the local density approximation. It was later found that the SMA was broken in the ground state of a condensate confined in a harmonic trap even in a homogenous field [13]. Nevertheless, the SMA continued to be used in studies of spinor condensates for its simplicity and validity in a broad range of experimental situations [11,15], in particular when the condensate size is smaller than the spin healing length, which determines the minimum domain size. On the other 
hand, the dynamical instability, leading to the spontaneous formation of dynamic spin domains, was found to occur in large ferromagnetic condensates prepared in excited initial states [9,16-18], while no such phenomenon was predicted to occur [16] or observed [19] in antiferromagnetic condensates. Similar instabilities were found in the transport of both types of spin-1 condensates in optical lattices [20]. It seemed however that spin domains were only to be found in antiferromagnetic condensates in the presence of inhomogeneous magnetic fields [2] or trapping potentials.

In this work, first we overview the results of our recent works $[21,22]$ and discuss the structure of spin-1 BoseEinstein condensates in the presence of a homogenous magnetic field. We show that the translational symmetry of a homogenous BEC is spontaneously broken and phase separation occurs in magnetized polar condensates if the magnetic field is strong enough. An analogous phenomenon has been predicted and observed previously in binary condensates [23,24]. In contrast, the cases when domain formation is driven by inhomogeneous external potentials or magnetic field gradients may be referred to as potential separation according to the naming used in Ref. 24. Here, we show that for a range of experimental conditions, it is energetically favorable for the system to consist of two separate phases composed of different stationary states. Finally, we demonstrate numerically that this phenomenon can be observed in a polar condensate trapped in a harmonic optical potential.

\section{Model}

We consider dilute spin-1 BEC in a homogenous magnetic field pointing in the $z$-direction. The mean-field Hamiltonian of this system is given by the expression,

$H=\int d \mathbf{r} \sum_{j=-, 0,+}\left(\frac{-\hbar^{2}}{2 M} \nabla \psi_{j}^{*} \nabla \psi_{j}+\frac{c_{0}}{2} n\left|\psi_{j}\right|^{2}+V(\mathbf{r})\left|\psi_{j}\right|^{2}\right)+H_{a}$,

where $\psi_{-}, \psi_{0}, \psi_{+}$are the wavefunctions of atoms in magnetic sub-levels $m=-1,0,+1, M$ is the atomic mass, $V(\mathbf{r})$ is an external potential and $n=\sum n_{j}=\sum\left|\psi_{j}\right|^{2}$ is the total atom density. The asymmetric part of the Hamiltonian is presented as:

$$
H_{a}=\int d \mathbf{r}\left(\sum_{j=-, 0,+} E_{j} n_{j}+\frac{c_{2}}{2}|\mathbf{F}|^{2}\right)
$$

where $E_{j}$ is the Zeeman energy shift for state $\psi_{j}$ and the spin density is,

$$
\mathbf{F}=\left(F_{x}, F_{y}, F_{z}\right)=\left(\psi^{\dagger} \hat{F}_{x} \psi, \psi^{\dagger} \hat{F}_{y} \psi, \psi^{\dagger} \hat{F}_{z} \psi\right)
$$

where $\hat{F}_{x, y, z}$ are the spin matrices [25] and $\psi=$ $=\left(\psi_{+}, \psi_{0}, \psi_{-}\right)$. The nonlinear coefficients are given by $c_{0}=4 \pi \hbar^{2}\left(2 a_{2}+a_{0}\right) / 3 M$ and $c_{2}=4 \pi \hbar^{2}\left(a_{2}-a_{0}\right) / 3 M$, where $a_{S}$ is the $s$-wave scattering length for colliding atoms with total spin $S$. The total number of atoms and the total magnetization

$$
\begin{gathered}
N=\int n d \mathbf{r}, \\
\mathcal{M}=\int F_{z} d \mathbf{r}=\int\left(n_{+}-n_{-}\right) d \mathbf{r},
\end{gathered}
$$

are conserved quantities. The Zeeman energy shift for each of the components, $E_{j}$ can be calculated using the BreitRabi formula [26]

$$
\begin{gathered}
E_{ \pm}=-\frac{1}{8} E_{H F S}\left(1+4 \sqrt{1 \pm \alpha+\alpha^{2}}\right) \mp g_{I} \mu_{B} B, \\
E_{0}=-\frac{1}{8} E_{H F S}\left(1+4 \sqrt{1+\alpha^{2}}\right)
\end{gathered}
$$

where $E_{H F S}$ is the hyperfine energy splitting at zero magnetic field, $\alpha=\left(g_{I}+g_{J}\right) \mu_{B} B / E_{H F S}$, where $\mu_{B}$ is the Bohr magneton, $g_{I}$ and $g_{J}$ are the gyromagnetic ratios of electron and nucleus, and $B$ is the magnetic field strength. The linear part of the Zeeman effect gives rise to an overall shift of the energy, and so we can remove it with the transformation

$$
H \rightarrow H+(N+\mathcal{M}) E_{+} / 2+(N-\mathcal{M}) E_{-} / 2 .
$$

This transformation is equivalent to the removal of the Larmor precession of the spin vector around the $z$-axis [21]. We thus consider only the effects of the quadratic Zeeman shift. For sufficiently weak magnetic field we can approximate it by $\delta E=\left(E_{+}+E_{-}-2 E_{0}\right) / 2 \approx \alpha^{2} E_{H F S} / 16$, which is always positive.

The asymmetric part of the Hamiltonian (2) can now be rewritten as

$$
H_{a}=\int d \mathbf{r}\left(-\delta E n_{0}+\frac{c_{2}}{2}|\mathbf{F}|^{2}\right)=\int d \mathbf{r} n e(\mathbf{r}),
$$

where the energy per atom $e(\mathbf{r})$ is given by [11]

$$
\begin{gathered}
e=-\delta E \rho_{0}+\frac{c_{2} n}{2}|\mathbf{f}|^{2}=-\delta E \rho_{0}+\frac{c_{2} n}{2}\left(\left|\mathbf{f}_{\perp}\right|^{2}+m^{2}\right), \\
\left|\mathbf{f}_{\perp}\right|^{2}=2 \rho_{0}\left(1-\rho_{0}\right)+2 \rho_{0} \sqrt{\left(1-\rho_{0}\right)^{2}-m^{2}} \cos \theta .
\end{gathered}
$$

We express the wavefunctions as $\psi_{j}=\sqrt{n \rho_{j}} \exp \left(i \theta_{j}\right)$ where the relative densities are $\rho_{j}=n_{j} / n$. We also introduced the relative phase $\theta=\theta_{+}+\theta_{-}-2 \theta_{0}$, spin per atom $\mathbf{f}=\mathbf{F} / n$, and magnetization per atom $m=f_{z}=\rho_{+}-\rho_{-}$. The perpendicular spin component per atom is $\left|\mathbf{f}_{\perp}\right|^{2}=f_{x}^{2}+f_{y}^{2}$.

The Hamiltonian (1) generates the Gross-Pitaevskii equations describing the mean-field dynamics of the system

$$
\begin{aligned}
& i \hbar \frac{\partial \psi_{ \pm}}{\partial t}=\left[\mathcal{L}+c_{2}\left(n_{ \pm}+n_{0}-n_{\mp}\right)\right] \psi_{ \pm}+c_{2} \psi_{0}^{2} \psi_{\mp}^{*}, \\
& i \hbar \frac{\partial \psi_{0}}{\partial t}=\left[\mathcal{L}-\delta E+c_{2}\left(n_{+}+n_{-}\right)\right] \psi_{0}+2 c_{2} \psi_{+} \psi_{-} \psi_{0}^{*},
\end{aligned}
$$

where $\mathcal{L}$ is given by $\mathcal{L}=-\hbar^{2} \nabla^{2} / 2 M+c_{0} n+V(\mathbf{r})$. 
By comparing the kinetic energy with the interaction energy, we can define a characteristic healing length $\xi=2 \pi \hbar / \sqrt{2 M c_{0} n}$ and spin healing length $\xi_{s}=2 \pi \hbar / \sqrt{2 M c_{2} n}$. These quantities give the length scales of spatial variations in the condensate profile induced by the spin-independent or spin-dependent interactions, respectively. Analogously, we define magnetic healing length as $\xi_{B}=2 \pi \hbar / \sqrt{2 M \delta E}$.

In real spinor condensates, the $a_{0}$ and $a_{2}$ scattering lengths have similar magnitude. The spin-dependent interaction coefficient $c_{2}$ is therefore much smaller than its spin-independent counterpart $c_{0}$. For example, this ratio is about 1:30 in a ${ }^{23} \mathrm{Na}$ condensate and 1:220 in a ${ }^{87} \mathrm{Rb}$ condensate far from Feshbach resonances [27]. As a result, the excitations that change the total density require much more energy than those that keep $n(\mathbf{r})$ close to the ground state profile. In our considerations we will assume that the amount of energy present in the system is not sufficient to excite the high-energy modes, and we will treat the total atom density $n(\mathbf{r})$ as a constant.

\section{Condensate without a trapping potential}

The ground states of spin- 1 condensates in homogenous magnetic field have been studied in a number of previous works $[2,13,28]$. The most common procedure [2] involves minimization of the energy functional with constraints on the number of atoms $N$ and the total magnetization $\mathcal{M}$. The resulting Lagrange multipliers $p$ and $q$ serve as parameters related to the quadratic Zeeman shift $\delta E$ and the magnetization $m$. An alternative method, elaborated in [13], consists of minimization of the energy functional in the parameter space of physically relevant variables $B$ and $m$. Most of the previous studies, however, were assuming that the condensate remains homogenous and well described by the single-mode approximation; in particular, the spatial structure observed in [2] resulted from the applied magnetic field gradient, but the BEC was assumed to be well described by the homogenous model at each point in space (local density approximation). In Ref. 13, the breakdown of the single-mode approximation was shown numerically for a condensate confined in a harmonic potential.

We correct the previous studies by showing that when the condensate size is larger than the spin healing length $\xi_{s}$, the translational symmetry is spontaneously broken and phase separation occurs in magnetized polar condensates if the magnetic field is strong enough. This phenomenon takes place when the energy of the spin state with the lowest energy is a concave function of $m$ for a given $\delta E$. On the contrary, the energy is always a convex function of $m$ for the ferromagnetic condensate, and no phase separation occurs. Note that phase separation has been previously predicted in binary condensates $[23,24]$ and in ferromagnetic condensates at finite temperature [29].
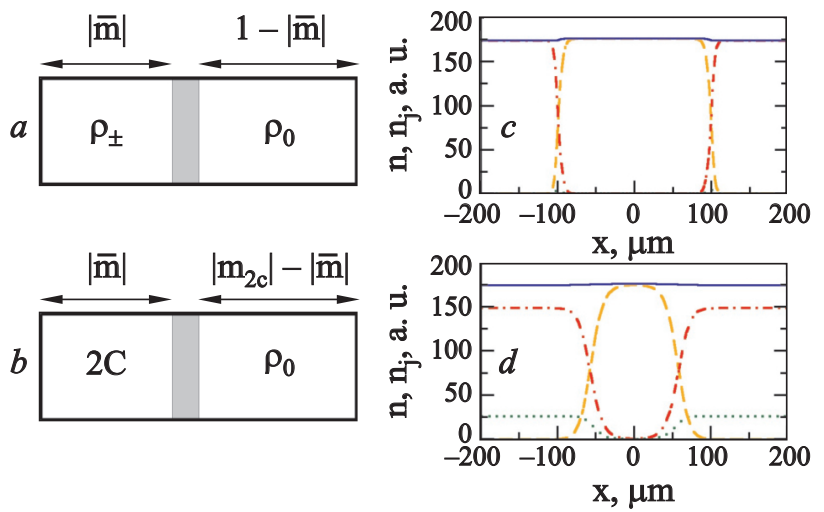

Fig. 1. Schematic structure of the phase separated states $\rho_{ \pm}+\rho_{0}$ (a) and $2 \mathrm{C}+\rho_{0}(b)$. The shaded region, in which all three components are nonzero, has the approximate extent of one spin healing length $\xi_{S}$ or magnetic healing length $\xi_{B}$, whichever is greater. The relative size of the domains is indicated with arrows. The corresponding wavefunction profiles obtained numerically with periodic boundary conditions in the case of ${ }^{23} \mathrm{Na}$ for $\bar{m}=0.5$ with $\delta E /\left(c_{2} n\right)=0.8(c)$ and $\delta E /\left(c_{2} n\right)=0.23(d)$. The $n_{+}, n_{0}$, and $n_{-}$components are depicted by dash-dotted, dashed, and dotted lines, respectively. The solid lines show the total density.

We construct ground states of the condensate using homogeneous stationary solutions of the GP equations (10)

$$
\psi_{j}(\mathbf{r}, t)=\sqrt{n_{j}} \mathrm{e}^{-i\left(\mu_{j}+\mu_{S}\right) t+i \theta_{j}},
$$

where $\mu_{S}=c_{0} n / \hbar$ is a constant and $\mu_{+}+\mu_{-}=2 \mu_{0}$ due to a phase matching condition. Following [22], we distinguish several types of stationary states. The states where only a single Zeeman component is populated $\left(n_{j}=1\right.$ for a specified $j=-, 0$, or + ) are named $\rho_{-}, \rho_{0}$, and $\rho_{+}$, respectively. The state where $n_{0}=0$ but $n_{-}, n_{+} \neq 0$ is the two-component $(2 \mathrm{C})$ state. The three-component states are classified according to the value of $\theta=\theta_{+}+\theta_{-}-2 \theta_{0}$. The states with $\theta=0$ are called phase-matched (PM) states, and the ones with $\theta=\pi$ are called anti-phase-matched (APM) states. For more details about this classification and the properties of the stationary states, refer to [22].

Two types of domain structures, depicted in Fig. 1, are composed of two different stationary states connected with a shaded region where all three components are nonzero. These two domain states have the advantage that the perpendicular spin is nonzero only in the transitory region, hence their energy is relatively low in polar condensates. In fact, these are the only phase separated states that can be the ground states of a homogenous condensate. Their energies per atom in the limit of infinite condensate size, which allows for neglecting of the relatively small intermediate region are

$$
e_{\rho_{ \pm}+\rho_{0}}=|\bar{m}| e_{\rho_{ \pm}}+(1-|\bar{m}|) e_{\rho_{0}},
$$




$$
e_{2 C+\rho_{0}}=\left.\frac{\bar{m}}{m_{2 C}} e_{2 C}\right|_{m=m_{2 C}}+\left(1-\frac{\bar{m}}{m_{2 C}}\right) e_{\rho_{0}},
$$

where $\bar{m}=\mathcal{M} / N$ is the average magnetization and the magnetization of the $2 \mathrm{C}$ component $m_{2 C}$ is a free parameter that has to be optimized to obtain the lowest energy state.

Table 1. Ground states of spin-1 condensates in homogenous magnetic field. The states $2 \mathrm{C}+\rho_{0}$ and $\rho_{ \pm}+\rho_{0}$ correspond to phase separation (see Fig. 1)

\begin{tabular}{c|c|c}
\hline \hline Condensate & Parameter range & Ground state \\
\hline \hline \multirow{3}{*}{ Ferromagnetic } & $2 \leq \frac{\delta E}{\left|c_{2}\right| n}$ and $\bar{m}=0$ & $\rho_{0}$ \\
$\frac{\delta E}{\left|c_{2}\right| n}<2$ or $\bar{m} \neq 0$ & PM \\
\hline \multirow{5}{*}{ Polar } & $\begin{array}{c}\bar{m}=0 \\
\frac{c_{2} n}{2} \leq \frac{\bar{m}^{2}}{2}\end{array}$ & $\rho_{0}$ \\
& $\frac{\delta E}{2}<\frac{1}{c_{2} n}$ and $\bar{m} \neq 0$ & $2 \mathrm{C}+\rho_{0}$ \\
$\frac{1}{2} \leq \frac{\delta E}{c_{2} n}$ and $\bar{m} \neq 0$ & $\rho_{ \pm}+\rho_{0}$ \\
\hline \hline
\end{tabular}

The ground states can be determined by comparing energies of the phase separated states with the energies of the homogenous solutions. The form of formulas (12) indicates that the phase separation will occur when the energy of the lowest homogenous state is a concave function of magnetization. The results for both polar and ferromagnetic condensates are collected in Table 1 . In the cases when no phase separation occurs, our results are in agreement with those obtained in [13]. Note that we assumed that the condensate size is much larger than $\xi_{s}$ and $\xi_{B}$. For small condensates, the results of [13] are correct.

In the case of high magnetic field strength, one of the Zeeman sub-levels is practically depleted [13] and the condensate becomes effectively two-component. The existence of the $\rho_{ \pm}+\rho_{0}$ phase in a polar condensate can then be understood within the binary condensate model $[23,24]$. We note that the experiment reported in Ref. 30, performed in this regime, can be viewed as the first confirmation of phase separation in spin-1 BEC in a homogenous magnetic field. However, the ground state was not achieved, and a multiple domain structure was observed.

In Fig. 2 we present the phase diagram of polar condensates, obtained both numerically and using analytical formulas from Table 1. The ground state profiles for a quasi$1 \mathrm{D}$ condensate were found numerically by solving the $1 \mathrm{D}$ version of Eqs. (10) [21]

$$
i \hbar \frac{\partial \tilde{\Psi}_{ \pm}}{\partial t}=\left[\tilde{\mathcal{L}}+\tilde{c}_{2}\left(\tilde{n}_{ \pm}+\tilde{n}_{0}-\tilde{n}_{\mp}\right)\right] \tilde{\Psi}_{ \pm}+\tilde{c}_{2} \tilde{\Psi}_{0}^{2} \tilde{\Psi}_{\bar{\mp}}^{*}
$$

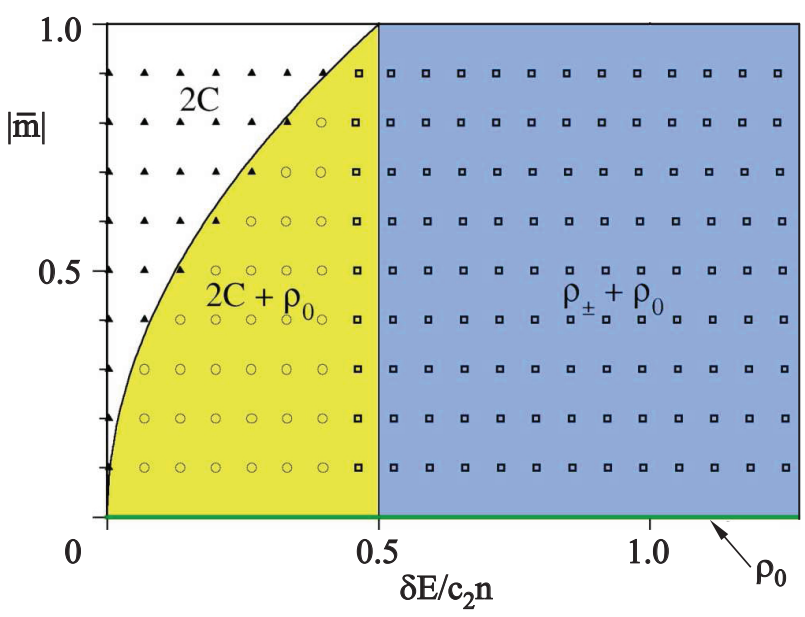

Fig. 2. Ground state phase diagram of the polar condensate. The symbols correspond to numerical data obtained for the parameters of ${ }^{23} \mathrm{Na}$, with solid triangles representing $2 \mathrm{C}$, open circles $2 \mathrm{C}+$ $+\rho_{0}$ and open squares $\rho_{ \pm}+\rho_{0}$. The solid lines and shading are given by the analytical formulas from Table 1 .

$$
i \hbar \frac{\partial \tilde{\psi}_{0}}{\partial t}=\left[\tilde{\mathcal{L}}-\delta E+\tilde{c}_{2}\left(\tilde{n}_{+}+\tilde{n}_{-}\right)\right] \tilde{\Psi}_{0}+2 \tilde{c}_{2} \tilde{\Psi}_{+} \tilde{\Psi}_{-} \tilde{\Psi}_{0}^{*},
$$

with $\tilde{\mathcal{L}}=-\left(\hbar^{2} / 2 m\right) \partial^{2} / \partial x^{2}+\tilde{c}_{0}$, where $\tilde{c}_{0}=4 \hbar \omega_{\perp}\left(2 a_{2}+a_{0}\right) / 3$, $\tilde{c}_{2}=4 \hbar \omega_{\perp}\left(a_{2}-a_{0}\right) / 3, \int d x \sum\left|\tilde{\psi}_{j}\right|=N$, and $\omega_{\perp}$ is the transverse trapping frequency. We imposed periodic boundary conditions on $\tilde{\psi}_{j}(x)$ and used the parameters corresponding to a ${ }^{23} \mathrm{Na} \mathrm{BEC}$ containing $N=5.2 \cdot 10^{4}$ atoms confined in a transverse trap with frequency $\omega_{\perp}=2 \pi \cdot 10^{3}$. The Fermi radius of the transverse trapping potential is smaller than the spin healing length, and the nonlinear energy scale is much smaller than the transverse trap energy scale, which allows us to reduce the problem to one spatial dimension [27,31]. The solutions were found numerically using the normalized gradient flow method [32,33], which is able to find a state which minimizes the total energy for given $N$ and $\mathcal{M}$, and fulfills the phase matching condition. The stability of the resulting states was verified using numerical time evolution according to Eqs. (13). The slight discrepancy between numerical and analytical results can be accounted for by the finite size of the condensate (the box size was $\sim 10 \xi_{s}$ ), and by the deviation from the assumption that the total density is constant (see the discussion at the end of Sec. 2). Due to the finite value of the ratio $c_{2} / c_{0}$ there is a slight density modulation, as is evident in Fig. 1,c,d.

\section{Condensate trapped in a harmonic optical potential}

The results from the preceding subsection can be verified experimentally in configurations involving toroidal or square-shaped optical traps [34]. However, in most experiments on BECs, harmonic potentials are used. The relevance of these results is not obvious in the case of harmonic trapping, since the coefficient $\delta E /\left(c_{2} n\right)$, one of the main 

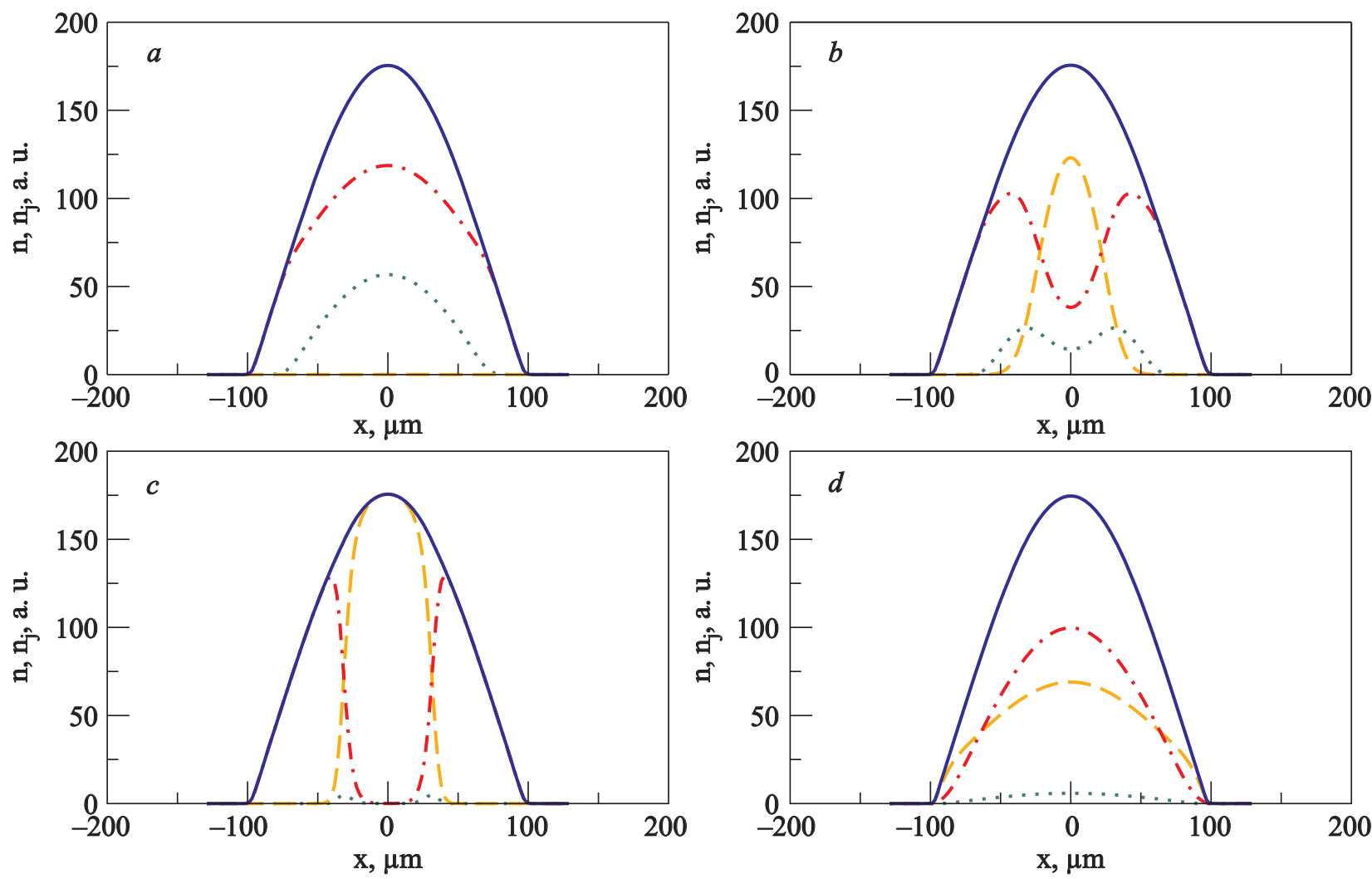

Fig. 3. Ground state profiles in a harmonic trap potential. Phase separation occurs in the polar ${ }^{23}$ Na condensate when the magnetic field strength is increased from $B=0.1 \mathrm{G}, \delta E /\left(c_{2} n_{\max }\right)=0.09 \quad(a)$ to $B=0.12 \mathrm{G}, \delta E /\left(c_{2} n_{\max }\right)=0.13 \quad(b)$ and $B=0.25 \mathrm{G}$, $\delta E /\left(c_{2} n_{\max }\right)=0.56(c)$. For comparison, the ground state of a ${ }^{87} \mathrm{Rb}$ condensate is shown in for $B=0.2 \mathrm{G}, \delta E /\left(c_{2} n_{\max }\right)=-0.41(d)$. The $n_{+}, n_{0}$, and $n_{-}$components are depicted by dash-dotted, dashed, and dotted lines, respectively. The solid lines shows the total density. Other parameters are $N=2.1 \cdot 10^{4}, \omega_{\|}=2 \pi \cdot 10\left({ }^{23} \mathrm{Na}\right), \omega_{\|}=2 \pi \cdot 7.6\left({ }^{87} \mathrm{Rb}\right), \omega_{\perp}=2 \pi \cdot 10^{3}$ and $\bar{m}=0.5$.

parameters controlling the condensate properties, varies in space due to the varying total density $n$.

The ground states in a highly elongated harmonic trap, where the parallel part of the potential has the form $V(x)=(1 / 2) M \omega_{\|}^{2} x^{2}$, are presented in Fig. 3 [33]. We can see that as the magnetic field strength is increased, phase separation occurs and the $\rho_{ \pm}+\rho_{0}$ domain state is formed. However, in contrast to the previous case, the transition is not sharp, and in particular there is no distinct $2 \mathrm{C}+\rho_{0}$ phase for any value of the magnetic field. Note that the state in Fig. 3,a is also spatially separated due to different Thomas-Fermi radii of the $\psi_{-}$and $\psi_{+}$components; however, this is an example of potential separation, as opposed to phase separation [24], since it is does not occur in the absence of the potential. On the other hand, Fig. 3,d shows that the components of ferromagnetic condensate are miscible even in the regime of strong magnetic field. In the regions where the wavefunctions overlap, the relative phase is equal to $\theta=0$ for ferromagnetic and $\theta=\pi$ for polar ground states, since these configurations minimize the spin energy (9).

The characteristic feature of phase separation in the polar BEC is that the $m=0$ domain tends to be localized in the center of the trap, as shown in Fig. 1,b and $c$. This can be explained by calculating the total asymmetric energy of the condensate (8), again assuming that the contribution from the intermediate region connecting the domains is negligible,

$$
\begin{aligned}
& H_{a} \approx \int_{\rho_{0}} d \mathbf{r} n(-\delta E)+\int_{\rho_{ \pm}} d \mathbf{r} n \frac{c_{2} n}{2}= \\
& =-\delta E(N-|\mathcal{M}|)+\frac{c_{2} N}{2}\langle n\rangle_{\rho_{ \pm}},
\end{aligned}
$$

where $\langle n\rangle_{\rho_{ \pm}}$is the mean condensate density within the area of the $\rho_{ \pm}$domain. We see that the energy will be the lowest if this domain is localized in the outer regions, where the condensate density is low.

\section{Generation of spin domains}

We propose a method for generation of spin domains described in the previous section by adiabatic switching of the magnetic field. We start with a condensate with all the atoms in the $m=1$ sublevel, in the ground state of a harmonic potential. Subsequently, some of the atoms are transferred to the $m=-1$ component in the rapid adiabatic passage process. The magnetic field is then suddenly switched off. In this way we can obtain a condensate with 

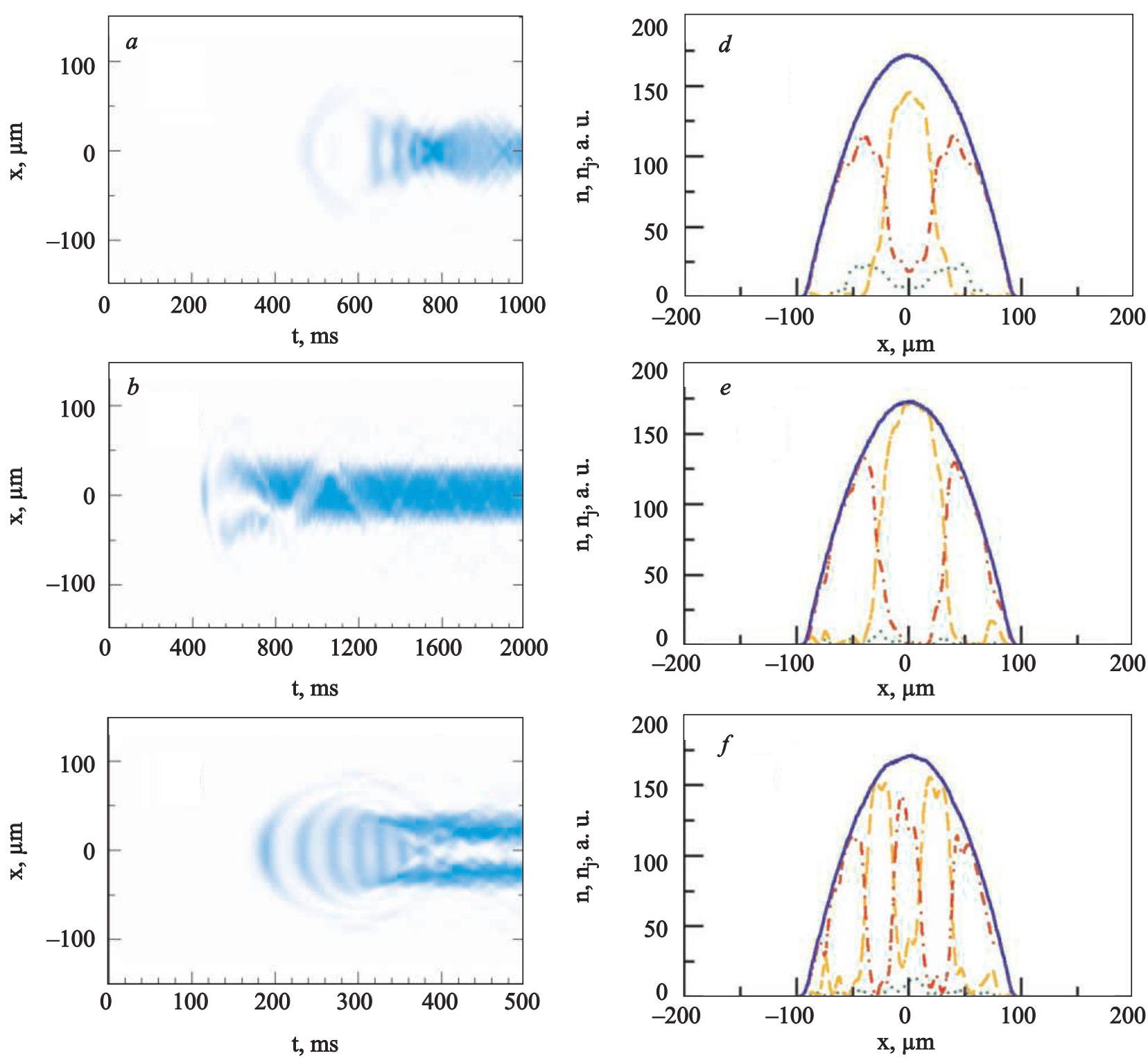

Fig. 4. Generation of spin domains by switching the magnetic field on. The magnetic field is gradually increased from zero to final value $B=0.15 \mathrm{G}$ during $t=1 \mathrm{~s}(a, d), B=0.25 \mathrm{G}$ during $t=2 \mathrm{~s}(b, e)$, and $B=0.25 \mathrm{G}$ during $t=0.5 \mathrm{~s}(c, f)$. The left column shows the time dependence of the atom density in the initially unoccupied $m=0$ component, and the right column shows the final domain profiles. In the last case, corresponding to nonadiabatic switching, multiple domains are formed. Other parameters are the same as in Fig. 3.

arbitrary magnetization in the $2 \mathrm{C}$ state, which is the ground state in $B=0$, see Fig. 2. Next, we gradually increase the magnetic field strength in an adiabatic process according to the formula

$$
B=\sqrt{\frac{t}{t_{\text {switch }}}} B_{\text {final }}
$$

where $t=0$ at the beginning of the switching process, $t_{\text {switch }}$ is the switching time, and $B_{\text {final }}$ is the desired final value of the magnetic field. The form of Eq. (15) assures that the quadratic Zeeman splitting grows linearly in time. We have confirmed that this condition improves the adiabaticity of the generation process. We present examples of the evolution of the condensate in Fig. 4. The left column shows the time dependence of the atom density in the initially unoccupied $m=0$ component, and the right column shows the final domain profiles. These should be compared with the ground state profiles in Fig. 3. The domains are generated for both the low and high magnetic field cases in times of the order of seconds, as shown in panels $(a, d)$ and $(b, e)$. However, when the switching time is significantly reduced, yielding the process no longer adiabatic, multiple metastable domains are formed as presented in panels $(c, f)$. This picture is in qualitative agreement with the experiment [30], where metastable spin domains were formed in nonadiabatic process within 50-100 ms. 


\section{Spin domains and dynamical stability}

Our results presented above show that the domain structure forming in polar condensates is absent in ferromagnetic BECs. This may seem to contradict the common understanding of ferromagnetism and the results of the quenched BEC experiment Ref. 9. The conventional picture of a ferromagnet involves many domains pointing in various directions separated by domain walls. Similar structure has been observed in Ref. 9. However, these cases correspond to the situations when there is an excess kinetic energy present in the system, due to finite temperature or excitation of the spatial modes. On the other hand, our study is limited to the ground states at $T=0$. It is easy to see from Eq. (9) that in zero magnetic field the ground state of a ferromagnetic BEC will always consist of a single domain with maximum possible value of the spin vector $|\mathbf{f}|=1$, pointing in the same direction at all points in space. However, when the temperature is finite, more domains can be formed each with a different direction of the spin vector.

We emphasize that the domain structure of the ground state in polar condensates is very different from the domains formed when the kinetic energy is injected in the system as in Ref. 9. The latter constantly appear and disappear in a random sequence $[9,16,17,21,35,36]$. On the contrary, the ground state domains are stationary and are positioned in the center of the trap. They exist in the lowestenergy state, while the dynamical domains require an amount of kinetic energy to be formed. The ground state domains can be prepared in an adiabatic process, involving adiabatic rf sweep or a slow change of the magnetic field $[30,35]$, while the kinetic domains require a sudden quench $[9,35]$.

The dynamical instability of ferromagnetic condensates that leads to spontaneous formation of spin domains has been investigated theoretically $[18,16,35]$ and observed in experiment [9]. An analogous phenomenon has been predicted recently for polar condensates in presence of magnetic field [21]. Here we correct the results of Ref. 21, by noting that the $\rho_{0}=1$ state is stable in ferromagnetic condensates for $\delta E>2\left|c_{2}\right| n$, and the $2 \mathrm{C}\left(\rho_{0}=0\right)$ state is stable in polar BECs if $\delta E<m^{2} / 2$. Both states become the ground states for these values of parameters. By investigating stability in various ranges of parameters, we are able to formulate a phenomenological law governing the dynamical stability of condensates: (i) The only stable state for both polar and ferromagnetic BECs in finite magnetic field is the ground state, as shown in Table 1; (ii) in zero magnetic field, the same is true for ferromagnetic condensates; However, all stationary states of polar condensates are dynamically stable in zero magnetic field [16,18,21]. The reason for the stability of polar condensates in vanishing magnetic field case is not yet clear. We note that the polar condensates in weak magnetic field may also be effectively stable on a finite time scale. As shown in Ref. 21, in this latter case the instability growth rate of unstable modes is proportional to the fourth power of the magnetic field strength. The time required for the development of instability may be much longer than the condensate lifetime [3].

\section{Conclusions}

We have studied the ground state of a spin-1 BEC in the presence of a homogenous magnetic field with and without an external trapping potential. We have found that without a trapping potential the translational symmetry can be spontaneously broken in polar BEC, with the formation of magnetic domains in the ground state. We have shown that these results may be used to understand the ground state structure in the presence of a trapping potential by mapping the locally varying density in the trap to the homogenous state. We have found that, depending on the magnetic field, the antiferromagnetic BEC ground state in the trap displays pronounced spin domains for a range of possible experimental conditions. Finally, we have discussed the relationship between the phenomenon of phase separation and the dynamical instability leading to the formation of dynamic spin textures.

\section{Acknowledgments}

This work was supported by the Australian Research Council through the ARC Discovery Project and Center of Excellence for Quantum-Atom Optics.

1. V.G. Baryakhtar, M.V. Chetkin, B.A. Ivanov, and S.N. Gadetskii, Dynamics of Topological Magnetic Solitons: Experiment and Theory, Springer-Verlag, Heidelberg (1994); and references therein.

2. J. Stenger, S. Inouye, D.M. Stamper-Kurn, H.-J. Miesner, A.P. Chikkatur, and W. Ketterle, Nature (London) 396, 345 (1998).

3. T.-L. Ho, Phys. Rev. Lett. 81, 742 (1998).

4. T. Ohmi and K. Machida, J. Phys. Soc. Jpn. 67, 1822 (1998).

5. M.-S. Chang, C.D. Hamley, M.D. Barrett, J. A. Sauer, K.M. Fortier, W. Zhang, L. You, and M. S. Chapman, Phys. Rev. Lett. 92, 140403 (2004).

6. A.E. Leanhardt, Y. Shin, D. Kielpinski, D.E. Pritchard, and W. Ketterle, Phys. Rev. Lett. 90, 140403 (2003).

7. D.M. Stamper-Kurn, M.R. Andrews, A.P. Chikkatur, S. Inouye, H.J. Miesner, J. Stenger, and W. Ketterle, Phys. Rev. Lett. 80, 2027 (1998).

8. M.S. Chang, Q.S. Qin, W.X. Zhang, L. You, and M.S. Chapman, Nat. Phys. 1, 111 (2005).

9. L.E. Sadler, J. M. Higbie, S.R. Leslie, M. Vengalattore, and D. M. Stamper-Kurn, Nature (London) 443, 312 (2006).

10. M. Vengalattore, J.M. Higbie, S.R. Leslie, J. Guzman, L.E. Sadler, and D.M. Stamper-Kurn, Phys. Rev. Lett. 98, 200801 (2007).

11. W. Zhang, D.L. Zhou, M.S. Chang, M.S. Chapman, and L. You, Phys. Rev. A72, 013602 (2005). 
12. J. Kronjäger, C. Becker, M. Brinkmann, R. Walser, P. Navez, K. Bongs, and K. Sengstock, Phys. Rev. A72, 063619 (2005)

13. W.X. Zhang, S. Yi, and L. You, New J. Phys. 5, 77 (2003).

14. S. Yi, Ö.E. Müstecaplioglu, C.P. Sun, and L. You, Phys. Rev. A66, 011601(R) (2002).

15. D.R. Romano and E.J.V. de Passos, Phys. Rev. A70, 043614 (2004).

16. W. Zhang, D.L. Zhou, M.-S. Chang, M.S. Chapman, and L. You, Phys. Rev. Lett. 95, 180403 (2005).

17. H. Saito, and M. Ueda, Phys. Rev. A72, 023610 (2005).

18. N.P. Robins, W. Zhang, E.A. Ostrovskaya, and Y.S. Kivshar, Phys. Rev. A64, 021601(R) (2001).

19. A.T. Black, E. Gomez, L.D. Turner, S. Jung, and P.D. Lett, Phys. Rev. Lett. 99, 070403 (2007).

20. J. Rustekoski and Z. Dutton, Phys. Rev. A76, 063607 (2007).

21. M. Matuszewski, T.J. Alexander, and Yu.S. Kivshar, Phys. Rev. A78, 023632 (2008).

22. M. Matuszewski, T.J. Alexander, and Yu. S. Kivshar, Phys. Rev. A80, 023602 (2009).

23. T.-L. Ho and V.B. Shenoy, Phys. Rev. Lett. 77, 3276 (1996); H. Pu and N.P. Bigelow, Phys. Rev. Lett. 80, 1130 (1998); M. Trippenbach, K. Goral, K. Rzazewski, B. Malomed, and Y.B. Band, J. Phys. B33, 4017 (2000).

24. E. Timmermans, Phys. Rev. Lett. 81, 5718 (1998).

25. T. Isoshima, K. Machida, and T. Ohmi, Phys. Rev. A60, 4857 (1999).
26. S. Wüster, T. E. Argue, and C.M. Savage, Phys. Rev. A72, 043616 (2005).

27. B.J. Dąbrowska-Wüster, E.A. Ostrovskaya, T.J. Alexander, and Y.S. Kivshar, Phys. Rev. A75, 023617 (2007).

28. F. Zhou, Phys. Rev. Lett. 87, 080401 (2001).

29. T. Isoshima, T. Ohmi, and K. Machida, J. Phys. Soc. Jpn. 69, 3864 (2000).

30. H.-J. Miesner, D.M. Stamper-Kurn, J. Stenger, S. Inouye, A.P. Chikkatur, and W. Ketterle, Phys. Rev. Lett. 82, 2228 (1999).

31. L. Salasnich, A. Parola, and L. Reatto, Phys. Rev. A65, 043614 (2002); W. Zhang and L. You, Phys. Rev. A71, 025603 (2005).

32. W. Bao and F.Y. Lim, SIAM J. Sci. Comput. 30, 1925 (2008).

33. F.Y. Lim and W. Bao, Phys. Rev. E78, 066704 (2008).

34. E.M. Wright, J. Arlt, and K. Dholakia, Phys. Rev. A63, 013608 (2000); K.E. Strecker, G.B. Partridge, A.G. Truscott, and R.G. Hulet, Nature 417, 150 (2002); S.K. Schnelle, E.D. van Ooijen, M.J. Davis, N.R. Heckenberg, and H. Rubinsztein-Dunlop, Opt. Express 16, 1405 (2008).

35. H. Saito, Y. Kawaguchi, and M. Ueda Phys. Rev. A75, 013621 (2007).

36. J. Mur-Petit, M. Guilleumas, A. Polls, A. Sanpera, M. Lewenstein, K. Bongs, and K. Sengstock, Phys. Rev. A73, 013629 (2006). 